\title{
THE ASSOCIATION OF CEREBRAL ANGIOMAS WITH INTRACRANIAL ANEURYSMS
}

\section{BY}

\section{J. S. BOYD-WILSON}

From the X-ray Department, Wellington Hospital, New Zealand

The symptoms separately associated with both intracranial aneurysms and angiomas are well understood and their diagnosis now presents no special problem. Rarely the two lesions may coexist, sometimes with confused symptoms, or, when intracranial haemorrhage is the presenting symptom, with the precise source of haemorrhage in doubt. Walsh and King (1942) recorded the first case of an associated angioma and aneurysm, since when 13 further examples have appeared in the literature.

In this paper three new cases are reported and the previous literature reviewed; a distinction is made between a true arterial aneurysm coexisting with an angioma, and the aneurysmal sac which is part of the malformation itself. Cases in the latter category are excluded. Suggestions are made as to the nature of the association of the two lesions, and the clinical significance of their association described. Case 1, which presented with subarachnoid haemorrhage, is of special interest because arteriography clearly demonstrated which lesion had bled.

\section{Case Reports}

- Case 1.-A married woman, aged 38, was admitted to the Hutt Hospital on September 6, 1957, under the care of Dr. R. Bakewell. Since the age of 12 she had had occasional left frontal headaches, usually on waking. On the morning of her admission she awoke with a headache which she took at first to be the usual migraine attack; on this occasion, however, the headache was occipital rather than above her left eye and her neck was stiff. The patient took some aspirin and left home to do some shopping; while waiting her turn to be served she suddenly lost consciousness.

On admission she complained of headache and neck stiffness but showed no localizing signs; lumbar puncture disclosed a heavily bloodstained fluid. Gradually headache became less severe and on the twelfth dayo bilateral carotid arteriography was performed. On then right side an aneurysm measuring $1.8 \mathrm{~cm}$. in diameter was present on the pericallosal artery, the calibre of which, for $1 \mathrm{~cm}$. proximal to the aneurysm, was reduced (Fig. 1a). The pericallosal artery was displaced $1 \mathrm{~cm} \Omega$ to the left of the mid-line. In the left posterior parieta region an angioma was present, supplied by the lef anterior and middle cerebral arteries (Fig. 1b).

The apparent spasm of the parent pericallosal vessel suggested that the source of haemorrhage was the aneurysm rather than the angioma; the patient was transferred to the Neurosurgical Unit, Dunedin Hospital, where at operation on September 26 a small intracerebral haemorrhage was found in the vicinity of the aneurysm

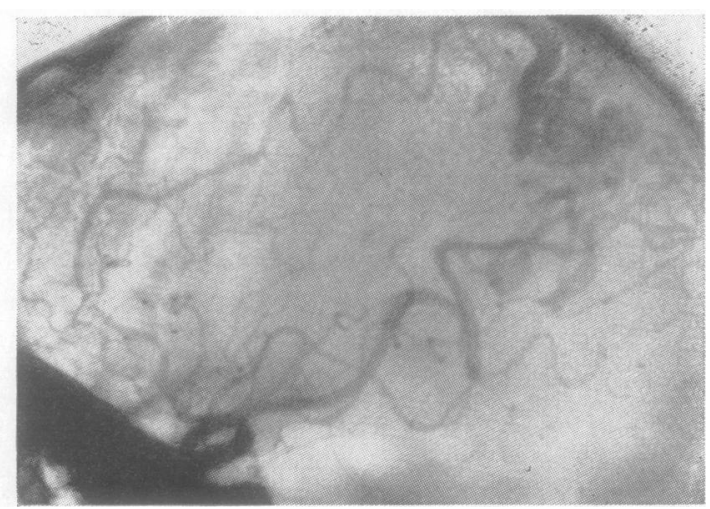

FIG. 1a and b.-Case 1: Bilateral carotid arteriograms showing an aneurysm $1.8 \mathrm{~cm}$. in diameter on the right pericallosal artery and a medium-sized angioma in the left posterior parietal region. The calibre of the pericallosal artery for $1 \mathrm{~cm}$. proximal to the aneurysm is markedly reduced. 


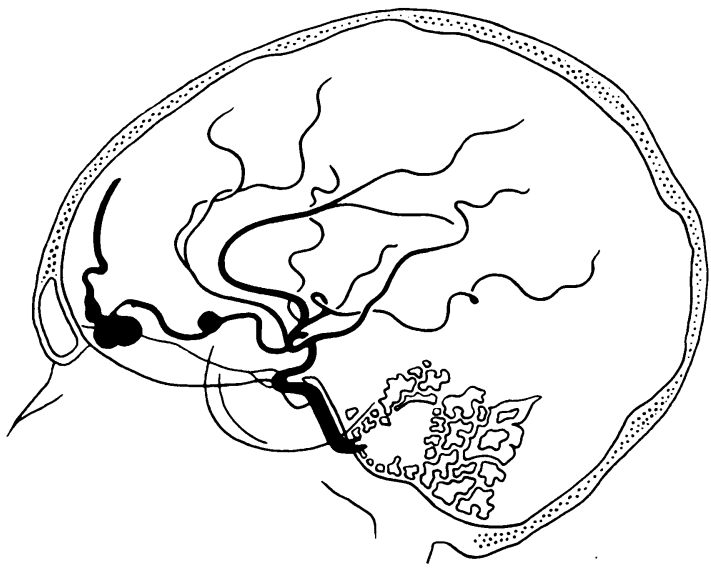

FIG. 2.-Case 2: A small angioma at the anterior extremity of the right frontal lobe with an arterial aneurysm on its feeding vessel.

and in turn communicated with a subdural haematoma. Both left and right anterior cerebral arteries were clipped and the subdural haematoma evacuated.

Case 2.-A man of 49, a scrub-cutter, was at work when he suffered sudden bifrontal headache; he fell to his knees but did not lose consciousness. Almost immediately he noticed neck stiffness but after a short interval was able to walk the three miles out of the bush. He was admitted that day, November 26, 1951, to the Wanganui Hospital under the care of Dr. C. P. Powles.

On admission the diagnosis was established by finding heavily bloodstained fluid on lumbar puncture; there were no localizing signs. His headache gradually abated and he was free of symptoms when on December 13 he was transferred to the Palmerston North Hospital for further investigation. A right carotid arteriogram on December 20 showed an aneurysm, $7 \mathrm{~mm}$. in diameter, on the fronto-polar branch of the right anterior cerebral artery; distal to the aneurysm the dilated fronto-polar artery continued to the anterior extremity of the frontal lobe to supply a small angioma (Fig. 2). Arteriography was not performed on the left side. The right common carotid artery was partially ligated on December 24 and ligation was completed three days later without incident. He was discharged home on December 31.

On February 19, 1952, the patient was admitted to the Neurosurgical Unit, Dunedin Hospital, and a right frontal craniotomy performed eight days later: the aneurysm was trapped and that part of the right frontal lobe containing the angioma excised.

Case 3.-A 49-year-old housewife was admitted to the Auckland Hospital under the care of Dr. E. G. Sayers on December 10, 1957, for the investigation of attacks of paraesthesiae and shaking in the left hand of 20 years' duration. Each attack began with a feeling of tingling in the left fingers, shortly followed by a twitching movement of flexion of the fingers and sometimes by

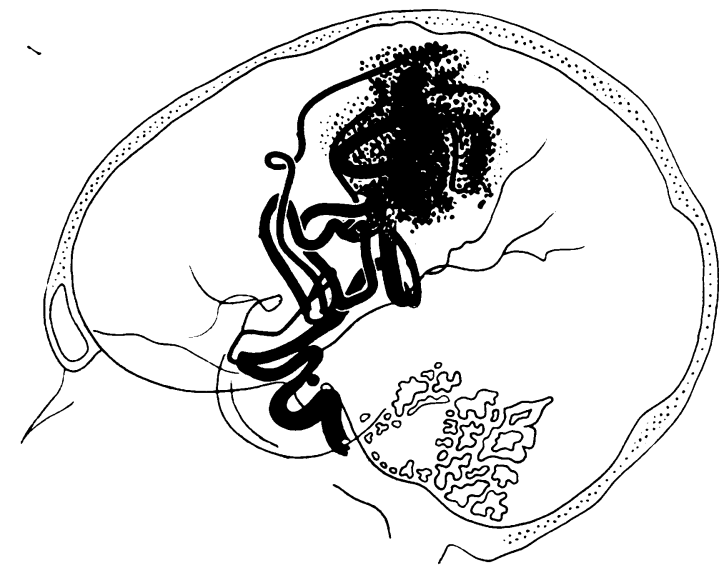

FIG. 3.-Case 3: A large angioma in the right upper parietal region, fed by markedly dilated and tortuous middle cerebral vessels; from the internal carotid artery in the region of the posterior communicating artery, a small aneurysm projects downward and backward.

blurring of vision; these symptoms lasted five to 10 minutes and for many years were followed by severe headache and vomiting. During the preceding two years she noticed a slowly progressive weakness and clumsiness of the left hand, although during this period the headache following each attack was less prominent. There was no family history of migraine.

On examination, a loud bruit was audible in both orbits and the right parietal region; apart from this the relevant physical findings were confined to the left arm. The left hand was noticeably smaller than the right, and there was a slight to moderate degree of generalized weakness of the extensor muscle groups and of the hand grip; the tendon reflexes in the left arm were brisker than those on the right side.

All laboratory investigations, including examination of the cerebrospinal fluid, were normal. An electroencephalogram showed a slightly asymmetrical voltage on the two sides with an otherwise normal background rhythm, the voltage on the right side being slightly less than that on the left.

No abnormality was present in the plain films of the skull. A right carotid arteriogram showed a large angioma in the right parietal region receiving its main blood supply from a markedly dilated middle cerebral artery; the internal carotid artery was also dilated. The anterior cerebral artery did not fill. At the junction of the internal carotid and posterior communicating arteries, a small aneurysm, measuring $3 \mathrm{~mm}$. in diameter, was present (Fig. 3).

The patient was treated conservatively.

\section{Review of Reported Cases}

Thirteen case reports of a cerebral angioma associated with one or more intracranial aneurysms have been found in the literature. Some reports were necessarily brief and the clinical or pathological details incomplete; in these, further information 
has been supplied by the original authors and is included in the following descriptions.

Walsh and King (1942) reported the first example of an associated aneurysm and angioma. Their patient, a Negro of 50, presented with intracranial haemorrhage. For four years previously he had suffered from intermittent right frontal headache and, from the first attack, a partial right third-nerve palsy. A clinical diagnosis of an aneurysm on the right side of the circle of Willis was made and at operation a large right supraclinoid aneurysm was found; it was, however, unruptured. The patient died several days later and at necropsy a left occipital angioma was revealed as the source of haemorrhage; a further small unruptured supraclinoid aneurysm was noted on the left side. Dandy (1944) later described the same case.

Arieti and Gray (1944) reported a further case, a man of 59 with a long history of epilepsy, disorderly behaviour, dypsomaniac attacks, and progressive mental deterioration. Necropsy revealed a left parietal angioma and a large left supraclinoid carotid aneurysm; in addition there were diffuse changes involving the subarachnoid vessels, similar to those in the angioma itself. Another aneurysm was present on the abdominal aorta.

Aring (1945) described the case of a woman of 30 who, when a child, began to have occasional minor fits and developed a left hemiparesis. She finally presented with multiple cranial nerve palsies and symptoms suggesting subarachnoid haemorrhage, dying four weeks after the onset. At necropsy an angioma occupied almost the whole of the right cerebral hemisphere; there were also unruptured aneurysms on the left posterior communicating, basilar, and anterior communicating arteries. Although on the fifth day the cerebrospinal fluid was grossly bloodstained, there was no evidence of intracranial haemorrhage post mortem. It may be presumed that the angioma was the source of haemorrhage.

Sugar (1951) successfully removed at operation both a large aneurysm and an angioma closely related to it but gave no details.

Laur (1953) reported in detail the history of a boy of 15 who initially presented with a right proptosis; a bruit was then audible over the whole of the left side of the skull. He shortly developed pulsating exophthalmos, frequent headache, and epilepsy. Arteriography disclosed a large left cerebral hemisphere angioma fed by an anomalous vertebral artery; on the right side a saccular aneurysm was present in the cavernous sinus, and two further aneurysms in the region of the anterior communicating artery also filled from this side. He was treated conservatively.
Hutton (1954) reported the next case, that of a man of 62, who, after a typical subarachnoid haemorrhage, was found on arteriography to have a left frontal angioma and an anterior communicating aneurysm which filled from the right side; the comment was made that in view of the impossibility of deciding which of the two lesions had bled, surgical treatment was considered inadvisable.

Walton $(1956,1958)$ described the case of a man of 48 who suffered a catastrophic subarachnoid haemorrhage. On admission to hospital within a few hours of the ictus he was deeply comatose with severe neck stiffness but no neurological signs; he deteriorated rapidly and died within 24 hours. At necropsy there was massive intraventricular and subarachnoid bleeding; a small angioma, $2 \mathrm{~cm}$. in diameter, was found close to the wall of the posterior horn of the right lateral ventricle. There was also an unruptured aneurysm, $1 \mathrm{~cm}$. in diameter, on the left posterior cerebral artery.

Paterson and McKissock $(1956,1958)$ described three cases. Their first was a man of 36 with a history of fits during the previous year; he showed no neurological signs. Arteriography demonstrated a large angioma at the left Sylvian point witho multiple small aneurysms on the keft anterior and middle cerebral arteries. He subsequently died from intracranial haemorrhage; no necropsy was performed.

Their second patient was a woman of 40 with a history of subarachnoid haemorrhage on three previous occasions. Arteriography disclosed a large right parietal angioma and two aneurysms on the dilated right internal carotid artery. She was treated conservatively and, three years later, remained well.

The third patient, a man of 54, died from a ruptured aneurysm found at necropsy to be situated on the right posterior inferior cerebellar artery $1.5 \mathrm{~cm}$. from its origin; he also had had an episode of intracranial haemorrhage one year previously. An angioma was present in the left cerebellar hemisphere. Paterson (1957) described the same case.

Kremer $(1957,1958)$ reported two cases. The first was a woman, aged 48 , with a history of episodic right-sided headache and left-sided sensory epileptic attacks since the age of 17 ; there was a further history of subarachnoid haemorrhage six weeks before admission. A loud bruit was heard over the scalp, especially to the right of the mid-line where there was a small cirsoid aneurysm, and a left homonymous hemianopic field defect was present with sensory impairment over the left side of the body. A right carotid arteriogram performed four years previously had revealed a large right parietal 
angioma, and a left carotid arteriogram six weeks before admission had shown that the angioma extended across the mid-line. Following admission a vertebral arteriogram was performed and the angioma was seen to involve as well the territory of the right posterior cerebral artery. An aneurysm was present at the bifurcation of the basilar artery.

The second case was a man, aged 27 , with a history of three generalized epileptic attacks at the age of 21 and two further attacks five years later. For two years he complained of left-sided headache and was finally admitted with a typical subarachnoid haemorrhage. Bilateral carotid arteriography showed an angioma at the left frontal pole fed by both internal carotid arteries, and an aneurysm on the anterior communicating artery which mainly filled from the right side. The angioma was successfully excised and the neck of the aneurysm clipped.

Brihaye and Blackwood (1957) made a detailed anatomical study of a large angioma in the right hemisphere of a man of 53 who, since early childhood, had suffered attacks of twitching of the left limbs sometimes followed by loss of consciousness. The diagnosis was finally established by arteriography following an episode of intracranial haemorrhage. At necropsy the angioma was seen to be fed mainly by dilated right anterior and middle cerebral arteries; on the supraclinoid portion of the right internal carotid artery, which was also dilated, were two small thin-walled saccular aneurysms. A further small aneurysm arose from the supraclinoid portion of the left internal carotid artery which was itself of normal calibre, and another aneurysm was present in the region of its bifurcation. On the posterior aspect of the basilar bifurcation two more aneurysms were present: it was one of these which had ruptured.

\section{Discussion}

What is the nature of this association of cerebral angiomas with intracranial aneurysms? If it be accepted that cerebral angiomas are congenital vascular malformations, and that intracranial aneurysms at least develop on the basis of a congenital vascular deficiency, then the association of the two lesions may be an expression of multiple disorders of vascular development. Under such circumstances patients with angiomas might be expected to show a higher incidence of aneurysms than an unselected sample of the population; this, however, does not appear to be the case. Of several large series of cerebral angiomas, only one, that of Paterson and McKissock (1956), described coexisting aneurysms; and in only three of their 110 patients with cerebral angiomas were aneurysms demonstrated. There are, nevertheless, two previously reported cases (Arieti and Gray, 1944; Laur, 1953) in which multiple congenital abnormalities were clearly present; Arieti and Gray used the term "progressive multiform angiosis" to describe the findings in their case which is, however, probably unique.

Paterson and McKissock (1956) suggested one further explanation of the association of the two lesions: that the increased blood flow consequent upon the presence of the angioma might throw abnormal stresses on the cerebral vessels concerned and so predispose to aneurysm formation. The prime haemodynamic effect of any arteriovenous shunt is to lower vascular resistance, and angiomas are no exception to this rule; according to Poiseuille's law the flow rate through the feeding vessel increases, often in practice to as much as three times the normal value. If there is any compensatory increase in cardiac output there will be a further increase in flow rate not only through the angioma itself but also through the whole of the brain; this increment amounted to approximately $30 \%$ in the two cases reported by Shenkin, Spitz, Grant, and Kety (1948). It is difficult to believe, however, that increased flow rate per se can predispose to the formation of saccular aneurysms: a more likely factor is the accompanying arterial dilatation, particularly if this is due to simple stretching, for this must aggravate any pre-existing congenital medial defect. Dible (1958) denied that such stretching is a factor of importance in the dilatation of an artery transmitting blood against lowered peripheral resistance; he suggested that the main factor is one of hyperplasia and hypertrophy involving the cells of the vessel wall. As all these changes, particularly the dilatation, are more pronounced on the side of the angioma, it might be expected that coexisting aneurysms would be commoner on that side; analysis of the present material does not, however, support this hypothesis. When the aneurysm is situated on the feeding vessel itself, as in Case 2, it is nevertheless difficult to believe that the altered haemodynamics have not in some way predisposed to its formation.

If the association of intracranial aneurysms with cerebral angiomas cannot be explained as an expression of multiple disorders of vascular development or on the basis of altered haemodynamics, the most likely explanation is that of coincidence. There have been various estimates of the frequency with which intracranial aneurysms are discovered in necropsy material, the most recent being that of Chason and Hindman (1958) who in a planned survey of 2,786 consecutive necropsies found 137 patients with congenital aneurysms, an incidence of $4.9 \%$. This figure may be logically applied to patients with cerebral angiomas, almost $5 \%$ of whom should be found to harbour an intracranial aneurysm. That 


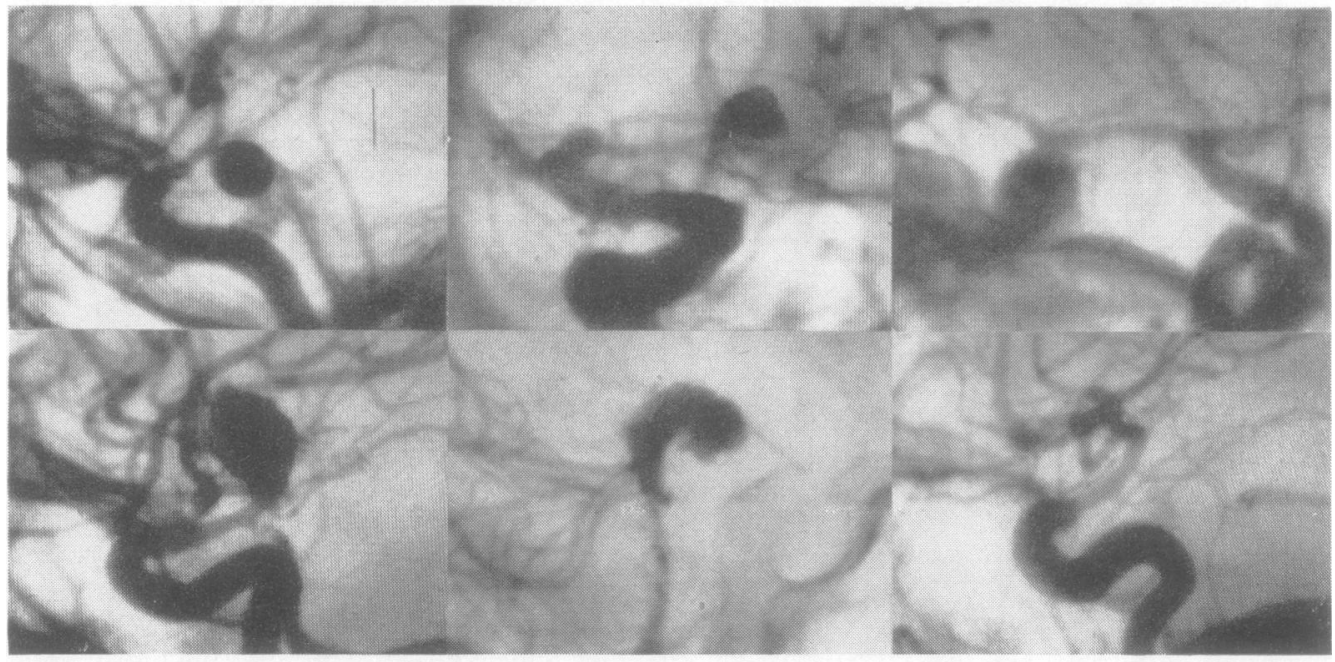

(d)

(e)

(f)

Fig. 4.-Typical examples of spasm involving the parent vessels of aneurysms in the following classical sites:- (a) Posterior communicating artery; (b) anterior communicating artery; (c) middle cerebral bifurcation; (d) internal carotid bifurcation; (e) basilar bifurcation; and (f) spasm of the internal carotid artery without any aneurysm demonstrated.

such an incidence has not been reported may be due to several factors. Thus, many supratentorial angiomas present with localizing signs and arteriography is then confined to the appropriate side; in few cases is vertebral arteriography as well as bilateral carotid arteriography performed. There are, moreover, certain inherent defects in the technique of cerebral arteriography whereby conventional anteroposterior and lateral views may fail to disclose very small aneurysms; this is probably a factor of some importance if the antero-posterior projection does not conform to certain standards (Boyd-Wilson, 1959).

The importance of the association of cerebral angiomas with intracranial aneurysms is twofold: the presence of both lesions may be responsible for confused symptoms, or, when intracranial haemorrhage is the presenting symptom, the precise source of haemorrhage may remain in doubt.

Analysis of the reported cases does not, however, suggest that confused symptoms are common; some such element is present in only three of the 15 cases in which a clinical history is available. There may be a history of a cranial nerve palsy due to the presence of the aneurysm, and the patient finally present with haemorrhage from the angioma, as in the case described by Walsh and King (1942); or there may be a history of epilepsy due to the angioma, and the patient present with haemorrhage from the aneurysm as in the case described by Brihaye and Blackwood
(1957). In neither case would a correct clinical diagnosis be possible. More suggestive is the develop- $-\frac{}{-}-$ ment of unexpected localizing signs as in Walsh $?$ and King's case, where, following a long history of을 right frontal headache and right third-nerve palsys on due to the large supraclinoid aneurysm on the sameo $\overrightarrow{0}$ side, the patient developed a right homonymous of hemianopia: the basis for this, an angioma in the left occipital lobe, was revealed at necropsy.

If surgery is contemplated in those cases which present with intracranial haemorrhage it is of importance to determine which lesion is the source of haemorrhage, but in no previously reported case was this possible. Thus Hutton (1954), describing his case, considered an attempt at surgical treatment inadvisable in view of the difficulty in deciding which of the two lesions had bled. Of the three cases reported in this paper, the first is of interest because arteriography clearly demonstrated the source of haemorrhage. In this case a segment of the pericallosal artery proximal to the aneurysm showed the arteriographic appearance of spasm; it was presumed that the aneurysm, rather than the angioma, had bled and this was confirmed at operation. The occurrence of spasm in the region of recently ruptured intracranial aneurysms was well described by Ecker and Riemenschneider (1951) who, using the strict criterion of a return to normal calibre on subsequent arteriography, were able to show evidence of spasm in six out of a total of 29 cases of 
surgically untreated subarachnoid haemorrhage. In their material the spasm was maximal at the site of the lesion and extended along the adjacent vessels for $1 \mathrm{~cm}$. or more.

Of 46 aneurysms recently demonstrated by arteriography in the Wellington Hospital, 36 (in 25 patients) were associated with subarachnoid haemorrhage; the calibre of the parent vessel of 16 of these aneurysms (in 14 patients) was reduced (Fig. 4a to e). Of the remaining nine aneurysms unassociated with subarachnoid haemorrhage, there was no similar appearance of the parent vessel in any case. Of 14 cases of subarachnoid haemorrhage in which no aneurysm or other lesion was disclosed by meticulous arteriography, one showed constriction of the supraclinoid portion of one internal carotid artery (Fig. 4f), and another constriction of both internal carotid arteries as well as the basilar artery. In all cases of subarachnoid haemorrhage, arteriography was performed percutaneously using 42 to $48 \%$ "urografin", the great majority under local anaesthesia at the end of the first week.

Johnson, Potter, and Reid (1958) drew attention to certain pitfalls in the arteriographic diagnosis of spasm and suggested that the radiological appearances might on occasion have a different basis: thus, arterial constriction due to true spasm induced by traction on supporting arachnoid bands, in turn due to distension of the subarachnoid space by blood, might be maintained by damage to the vascular wall or by the pressure of blood clot. They described one case where "spasm" was present at operation and appeared unchanged in the post-mortem arteriogram. In the present series, one of the 14 patients with evidence of spasm came to necropsy two days after the arteriogram: all the affected vessels were then of normal calibre. In this case the arteriographic appearances were undoubtedly due to the presence of true spasm.

It may be said, then, that arterial constriction is commonly associated with arteriographically demonstrated intracranial aneurysms; that it is usually the parent vessel which is so affected, not vessels at a distance from the aneurysm; that more than one aneurysm in the same patient may be involved; that the appearances are associated with ruptured and not unruptured aneurysms; and, occasionally, that similar changes may be present in cases of subarachnoid haemorrhage when no aneurysm is demonstrated. Whatever the real nature of this constriction (probably due to true spasm in the majority of cases) the fact remains that such a finding constitutes valid evidence of recent haemorrhage, and is a radiological sign of importance when the source of haemorrhage remains in doubt.

\section{Summary}

Three new cases of associated cerebral angioma and intracranial aneurysm are reported, and the literature reviewed. Suggestions are made as to the nature of this association, the clinical significance of which is seen more particularly in cases which present with intracranial haemorrhage, the origin of which may be obscure. In one of the cases reported apparent spasm of the parent vessel suggested that the aneurysm, rather than the angioma, was the source of haemorrhage and this proved to be the case at operation. Such narrowing of the parent vessel is shown to be a radiological sign of importance when arteriography discloses more than one lesion as a possible source of haemorrhage.

Acknowledgement is made to Dr. Stephen Moor and Dr. Gavin Glasgow, and to Mr. Anthony James, who kindly offered to make their cases available.

\section{REFERENCES}

Arieti, S., and Gray, E. W. (1944). Arch. Neurol. Psychiat. (Chicago), $51,182$.

Aring, C. D. (1945). Dis. nerv. Syst., 6, 119.

Boyd-Wilson, J. S. (1959). J. Neurosurg. 16, 297.

Brihaye, J., and Blackwood, W. (1957). J. Path. Bact., 73, 25.

Chason, J. L., and Hindman, W. M. (1958). Neurology, 8, 41.

Dandy, w. E. (1944). Intracranial Arterial Aneurysms. Comstock Publishing Co., Ithaca, New York.

Dible, J. H. (1958). Lancet, 1, 1031.

Ecker, A., and Riemenschneider, P. A. (1951). J. Neurosurg., 8, 660. Hutton, C. F. (1954). Brit. J. Radiol., 27, 471.

Johnson, R. J., Potter, J. M., and Reid, R. G. (1958). J. Neurol. Neurosurg. Psychiat., $21,68$.

Kremer, M. (1957). Proc. roy. Soc. Med., 50, 281.

(1958). Personal communication.

Laur, A. (1953). Disch. Arch. klin. Med., 200, 236.

Paterson, J. H. (1957). In Modern Trends in Neurology (second series), p. 105. Butterworth, London. and McKissock, W. (1956). Brain, 79, 233.

- , and Mckion.

Shenkin, H. A., Spitz., E. B., Grant, F. C., and Kety, S. S. (1948). J. Neurosurg., 5, 165.
.

Sugar, O. (1951). Ibid., 8, 3. Walsh, F. B., and King, A. B. (1942). Arch. Ophthal. (Chicago),

Walton, J. N. (1956). Subarachnoid Haemorrhage. Livingstone, Edinburgh and London.

- (1958). Personal communication. 